\title{
Evaluating the Effects of Online tDCS with Emotional $n$-back Training on Working Memory and Associated Cognitive Abilities
}

\author{
Gregory S. Berlin, Abel S. Mathew, Salahadin Lotfi, Ashleigh M. Harvey, and Han-Joo Lee* \\ University of Wisconsin-Milwaukee, Milwaukee, Wisconsin, USA
}

\begin{abstract}
Working memory (WM) is a core cognitive ability important for everyday functioning. A burgeoning area of research suggests that WM can be improved via working memory training (WMT) paradigms. Additionally, recent research has shown that WM may be enhanced through noninvasive neuromodulation such as transcranial direct current stimulation (tDCS). In this study, we evaluated how a single-session, brief-but-concentrated combination of tDCS over the left dorsolateral prefrontal cortex (dIPFC; F3 region), paired with a WMT paradigm utilizing emotional stimuli (emotional $n$-back) could produce gains in WM and associated, untrained cognitive abilities. Healthy undergraduate participants were randomized to receive either active tDCS and WMT, or sham-tDCS and WMT. Cognitive abilities (WM, attention control, and cognitive inhibition) were measured before and after the intervention. No significant differences were found in WM performance or associated abilities between those who received active or sham tDCS. Individuals in both groups evidenced a faster reaction time on an Operation Span task, and an Emotional Stroop Task, following the WMT session. These findings add to the mixed picture of the effectiveness of single-session WMT protocols and highlight the importance of the dose-response relationship in training core cognitive processes such as WM.
\end{abstract}

Keywords: tDCS; working memory training; $n$-back; dIPFC

Citation: Berlin, G. S., Mathew, A. S., Lotfi, S., Harvey, A. M., \& Lee, H.-J. (2020). Evaluating the effects of online tDCS with emotional $n$-back training on working memory and associated cognitive abilities. NeuroRegulation, 7(3), 129-140. https://doi.org/10.15540/nr.7.3.129

*Address correspondence to: Han-Joo Lee, PhD, 2441 Hartford Ave, GAR 211, Department of Psychology, University of WisconsinMilwaukee, Milwaukee, WI 53217, USA. Email: leehj@uwm.edu

Copyright: (c) 2020. Berlin et al. This is an Open Access article distributed under the terms of the Creative Commons Attribution License (CC-BY).

\section{Introduction}

Working memory (WM) is a cognitive ability with a limited capacity related to temporary storage and manipulation of information (Baddeley, 1992). WM is critically important when executing multiple tasks and informing behavior choices for upcoming events (D'Esposito \& Postle, 2015). Deficits in WM function have been linked to failure in real-world tasks (Beilock \& Carr, 2005) and in potentiating emotional problems such as depression and anxiety symptoms (Matsuo et al., 2007; Moran, 2016).

Recent research has suggested that WM is a malleable cognitive process that can be improved

\section{Edited by:}

Rex L. Cannon, PhD, SPESA Research Institute, Knoxville, Tennessee, USA

\section{Reviewed by:}

Rex L. Cannon, PhD, SPESA Research Institute, Knoxville, Tennessee, USA

Jon A. Frederick, PhD, Lamar University, Beaumont, Texas, USA Genomary Krigbaum, PsyD, Grand Canyon University, Phoenix, Arizona, USA 
posttraumatic stress disorder (Larsen et al., 2019; Owens, Koster, \& Derakshan, 2013; Sari, Koster, Pourtois, \& Derakshan, 2016; Schweizer et al., 2017). Additionally, emotional WMT paradigms have been shown to increase the efficiency of the frontoparietal cognitive control network that is critical for WM function (Schweizer, Grahn, Hampshire, Mobbs, \& Dalgleish, 2013; Schweizer, Hampshire, \& Dalgleish, 2011).

A promising means to further enhance WMT is to utilize a direct brain stimulation approach such as transcranial direct current stimulation (tDCS). tDCS is a noninvasive form of brain stimulation purported to alter cortical excitability (Nitsche et al., 2003). Meta-analyses suggest that tDCS can significantly enhance WM capabilities and reaction time on cognitive tasks when paired with WMT (Brunoni \& Vanderhasselt, 2014; Mancuso, llieva, Hamilton, \& Farah, 2016). Considerable evidence suggests that key components of WM function are housed in the frontal lobe structures of the brain, particularly within the dorsolateral prefrontal cortex (dIPFC; Barbey, Koenigs, \& Grafman, 2013; Eriksson, Vogel, Lansner, Bergström, \& Nyberg, 2015). As such, many studies utilizing tDCS to alter cortical excitability related to WM target the dIPFC (Plewnia, Schroeder, Kunze, Faehling, \& Wolkenstein, 2015; Ruf, Fallgatter, \& Plewnia, 2017; Schulze, Grove, Tamm, Renneberg, \& Roepke, 2019). Considering the ease of use and safety of the device (Bikson et al., 2016), tDCS and stimulation of the dIPFC has potential to augment the power of existing WMT programs.

Significant questions remain regarding the facilitative effect of tDCS on WMT, however. Firstly, there is mixed evidence regarding the necessary training threshold of pairing tDCS and WMT to produce cognitive gains. Some have suggested that a single session of tDCS can produce neurocognitive gains in WM (Fregni et al., 2005). However, others have suggested that tDCS, with or without WMT, needs repeated administration to induce long-term potentiation (Alonzo, Brassil, Taylor, Martin, \& Loo, 2012; Meinzer et al., 2014), yet others have suggested that tDCS has little effect on cognitive function at all (Horvath, Forte, \& Carter, 2015). Within the literature, the strength and duration of current delivery is also varied, but generally kept within 1 milliampere $(\mathrm{mA})$ administered for approximately 5 to 30 minutes with few studies providing stimulation on the upper end of this duration (Clarke, Browning, Hammond, Notebaert, \& MacLeod, 2014; Filmer, Lyons, Mattingley, \& Dux, 2017; Nitsche et al., 2003; Ruf et al., 2017). If a brief but concentrated tDCS-WMT training paradigm can produce changes in core cognitive capabilities, such an intervention can be easily transported and utilized in a variety of contexts where WM is impacted. Thus, if a single-session training with a concentrated electrical and cognitive dosage could produce a therapeutic signal, there would be grounds for further exploration of such an intervention.

Secondly, while most $n$-back training paradigms use nonemotional stimuli to train WM processes (Soveri et al., 2017), such WM processes are intricately tied to underlying emotional valence. For instance, memory processes for emotional stimuli may be enhanced due to connections between the amygdala and cortical regions (Dolcos, LaBar, \& Cabeza, 2004), and conversely diminished during aggravation of negative affective and anxiety states (Figueira et al., 2017; Moon \& Jeong, 2015). Recent work suggests that WMT programs can be enhanced by training WM processes using emotional stimuli (Larsen et al., 2019; Schweizer et al., 2013). Despite the potent link between WM and emotional content, very few studies utilize emotional WMT in conjunction with tDCS (Martin et al., 2018; Schmidt, Wolkenstein, \& Plewnia, 2015). Additional research is needed to explore how the combination of tDCS and emotional WMT can enhance cognitive training outcomes.

In this study, we sought to explore the usefulness of a single, concentrated, cognitive training paradigm in improving WM and associated cognitive abilities. To this end, we sought to amplify the tDCS-WMT paradigms used in previous studies by incorporating emotional valence in our cognitive training task and increasing the dosage of both WMT and tDCS in terms of time in the context of a single session. We hypothesized that individuals receiving anodal direct current stimulation in conjunction with emotionally valanced WMT would show improvements in both WM performance and transfer improvements in untrained but closely associated cognitive abilities such as attention control and cognitive inhibition.

\section{Method}

\section{Participants}

Forty-four $(n=44)$ undergraduate students between ages 18 and 60 were recruited from a university in the Midwestern United States. This study utilized a nonclinical student sample (healthy individuals) who were free of active psychiatric complaints, were not prescribed psychotropic medications, and did not report a history of head injuries or neurological 
complaints (e.g., history of seizures). A healthy sample was recruited for this study to factor out the deleterious effect of psychiatric comorbidities on WM processes (Lukasik, Waris, Soveri, Lehtonen, \& Laine, 2019; Salazar-Villanea, Liebmann, GarnierVillarreal, Montenegro-Montenegro, \& Johnson, 2015).

Of the 264 participants who completed the prescreening, 86 individuals passed the prescreening criteria and were invited to participate in a full eligibility (FE) assessment. Fifty-seven individuals consented to participation, and 47 of those met study entry criteria; 10 individuals were excluded for reasons such as metal implants, history of concussions, or active psychotropic medications. Forty-seven participants completed the FE, but three individuals withdrew participation prior to randomization. Full demographic and clinical information of our sample is found in Table 1. The experimental and placebo groups did not significantly differ from each other regarding gender or age, or in baseline characteristics such as cognitive performance, or baseline anxiety.

\section{Table 1}

Demographic and clinical characteristics of the study sample

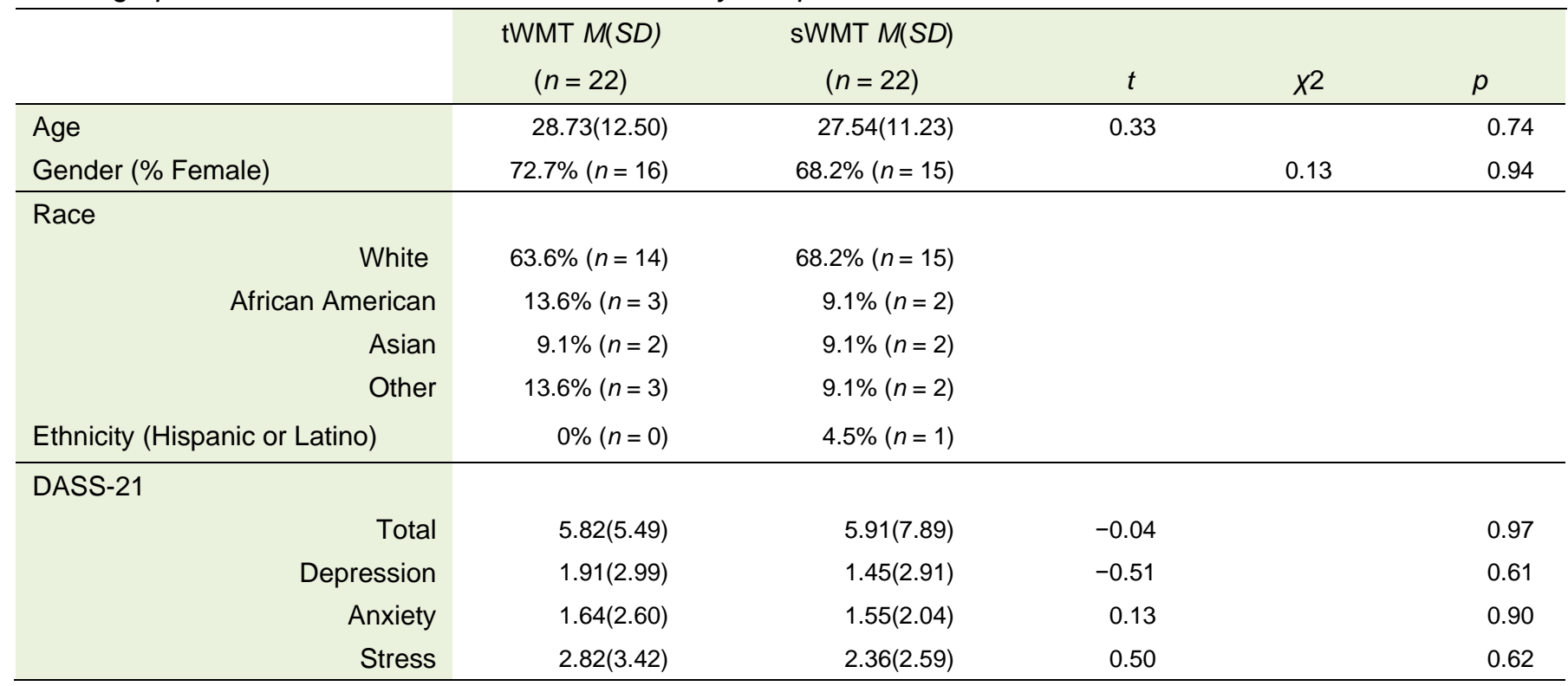

Note. $\mathrm{tWMT}=$ working memory training plus active direct current stimulation; sWMT = working memory training plus sham direct current stimulation; DASS-21 = depression, anxiety, and stress scale.

${ }^{*} p<.05 ;{ }^{* *} p<.01$

\section{Study Procedures}

A study flow-chart is found in Figure 1. Interested participants were invited to complete a prescreening survey including an online prescreening consent form, Diagnostic History Scale (DHS; a researchermade 14-item self-report measure to assess the presence of any comorbid psychiatric conditions or allergies that may affect study participation), and Safety Screening Questionnaire (SSQ; a 17-item self-report measure that asks about contraindications to tDCS stimulation). Those who did not report current psychiatric complaints, alcohol and substance use, history of concussions or head injuries, mental implants in head, history of seizure, epileptiform, or migraines, and concomitant psychotropic medications were invited to the FE session. Written informed consent was obtained in person at the laboratory visit prior to any study activities. All study procedures were approved by the university's Institutional Review Board (IRB).

The remainder of study procedures took place over a single session. Participants were first reassessed for eligibility criteria using the Mini International Neuropsychiatric Interview (MINI) version 6.0, DHS, and SSQ (semi-structured interview format) to ensure safety for participation. 


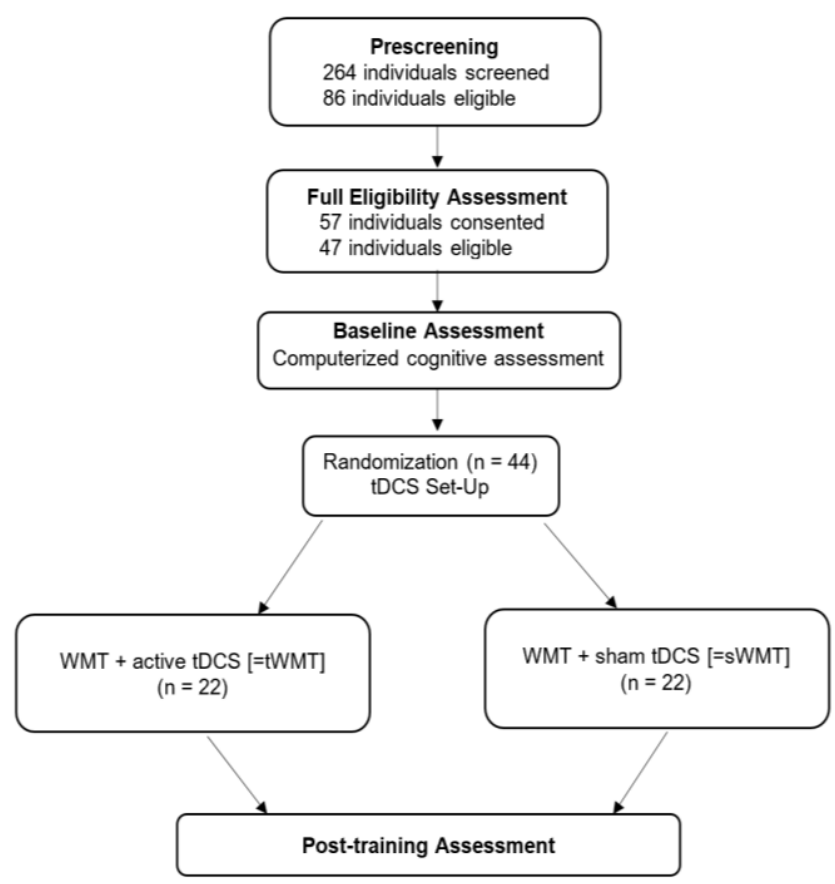

Figure 1. Flow of study procedures.

Following verification of study eligibility, participants were assessed using a battery of computerized cognitive tests to measure WM and attention. Cognitive function was measured at baseline (BL), and again following training (posttraining [PT]). The Depression Anxiety and Stress Scale (DASS-21) was used to measure depression, anxiety, stress, and general negative affect at BL (Lovibond \& Lovibond, 1995). While we targeted a healthy sample to factor out the effect of psychiatric comorbidity on cognitive function, we measured the general level of emotional symptomology in our sample to further identify any confounding effects of negative affect on cognitive function. Computerized cognitive assessment and training paradigms were presented using E-Prime 2.0 (Psychology Software Tools, Inc., Sharpsburg, PA), and the remainder of computerized assessment used Inquisit software (Millisecond Software, Seattle, WA). Questionnaires were administered using Qualtrics Survey Tools (Qualtrics, Provo, UT).

Randomization took place after $\mathrm{BL}$ assessment according to the following schedule: either WMT + active tDCS group (tWMT; $n=22$ ), or WMT + sham tDCS group (sWMT; $n=22$ ). Following randomization, the tDCS apparatus was attached, and participants completed 60 min of WMT. This training period was approximately two to three times the regular dose of one WMT session typically used in the literature (Larsen et al., 2019; Schweizer et al., 2013). Participants in the tWMT condition received $30 \mathrm{~min}$ of current stimulation while training WM processes, and participants in the sWMT only received $30 \mathrm{~s}$ of stimulation (ramp-up) to induce the feeling of stimulation (i.e., tingling). Stimulation started immediately upon trial 1 of the $n$-back paradigm. Thus, tWMT received online direct current for half of their training session, while sWMT participants essentially completed a 60-min memory training session without stimulation. Following WMT, participants repeated computerized cognitive assessment from $\mathrm{BL}$ to measure a pre-to-post change in memory and associated cognitive abilities.

\section{Computerized Cognitive Assessment}

Working memory (automated complex span tasks [ACST]). WM performance was the primary outcome measure in this study. WM was measured through the automated complex span tasks (ACST; Oswald, McAbee, Redick, \& Hambrick, 2015), which was comprised of three computerized span tasks to measure discrete aspects of WM processing. The operation span task prompted participants to solve a series of math operations while remembering a set of unrelated distractor numbers. The reading span task presented participants with sensical and nonsensical sentences (approximately 10-15 words long) and asked participants to differentiate which they were while also remembering the order of a string of digits. In the symmetry span task, participants were presented with a set of $8 \times 8$ matrices of black and white squares, and participants were instructed to judge whether the matrices were symmetrical or asymmetrical along a vertical line while also remembering the location of a red square positioned in a $4 \times 4$ matrix. Each span task provided two indices of WM function: absolute scores referred to the number of trials in which a participant recalled all target stimuli in order without error and relative scores referred to the proportion of correct responses.

WM training paradigms have also been shown to induce transfer effects on untrained cognitive tasks (Jaeggi et al., 2010), and WM has been shown to be critically important for overall allocation of attentional resources, especially in threat contexts (Stout, Shackman, Johnson, \& Larson, 2015; Stout, Shackman, \& Larson, 2013). Thus, we used the following tasks as secondary outcome measures to probe transfer effects of our training protocol:

Interference control (emotional Stroop task [EST]). The EST presented words from three distinct categories (neutral, positive, and negative) and prompted participants to select, with colored 
keys on the keyboard, the color of the word on the computer screen. The EST is useful in identifying interference effects in processing emotional stimuli, such that longer reaction times (RT) indicate greater difficulty in processing information effectively. The EST produced a general RT index for each word condition (e.g., Negative RT, Neutral RT, etc.), and a bias score for each word category by subtracting the neutral RT from the category RT (e.g., Negative Bias $=$ Negative RT - Neutral RT). Participants were tested with one practice block (20 trials) and one test block (75 trials; Smith \& Waterman, 2003).

Attentional control (attention network task [ANT]). We utilized the brief ANT to measure overall efficiency in attentional networks. Participants were presented with a fixation cross (400 ms), followed by five directional arrows arranged in a line. Participants responded quickly to identify the direction of the central arrow (left or right). The arrows flanking the central target either provided congruent information (e.g., all arrows were pointing in the same direction), or incongruent information (e.g., arrows flanked the central target with random left/right directions). Participants were tested with one practice block (12 trials), and three test blocks (48 trials each). The ANT provided three indices of attentional control: (1) alerting (achieving and maintaining alertness); (2) orienting (orienting attention to a specific location); and (3) conflict (resolving conflict between incongruent stimuli; Fan, McCandliss, Sommer, Raz, \& Posner, 2002).

\section{Computerized Working Memory Training}

WM was trained with an emotionally adaptive dual $n$ back task. This WMT involved a presentation of emotionally salient faces and words to target constant updating of information in WM and shifting between two different modes of stimuli (Larsen et al., 2019). In each trial, one of eight fearful faces (4 males and 4 females) was presented within a $3 \times 3$ grid while a negative spoken word (female voice; disaster, cancer, etc.) was simultaneously delivered. The $n$ refers to how many trials back from the current trial a participant must withhold in their working memory. Thus, to achieve a correct response, participants had to determine whether the location of a face and word stimuli presented in the current trial matched the face location and word presented $n$-trials back (e.g., 1-trial back, 2-trials back, etc.). The task is both a cognitive training tool and assessment modality in that practice on the task is thought to empower working memory abilities, and data from each session provides an index of working memory performance at that point in time. The task was adaptive, and training began at the 1-back level and progressed depending on performance through the entirety of the hour training session. Difficulty was raised (i.e., 2-back, 3-back, 4-back) contingent on performance accuracy over $95 \%$ for both modalities (i.e., faces and words). A performance accuracy of less than $75 \%$ lowered the difficulty level by 1 -back. The last level of $n$-back was carried across blocks so that performance was continuous. Though the task was adaptive, the $n$-back would not be classified as neurofeedback because participants were not encouraged to modify performance based on physiological readings. Training took place over four blocks (first block $=30 \mathrm{~min}$, blocks $2-4=10 \mathrm{~min}$ each).

tDCS placement and dosage. Following BL assessment and randomization, participants were offered a short break before attaching the tDCS apparatus. Electrode sponges were moistened with $0.9 \%$ saline solution and wrung out to release excess liquid. Participants were seated in front of the computer monitor, and the target area for stimulation (left forehead) was wiped with an alcohol wipe. Placement of the anodal (positive) electrode was over the F3 region to stimulate the dIPFC (Hill, Fitzgerald, \& Hoy, 2016). Electrodes were placed according to the 10-20 international positioning system using the positioning tool reported by Beam, Borckardt, Reeves, and George (2009). We utilized 2x2 Amrex electrodes. The stimulator used was the Chattanooga lonto dual channel electrophoresis system (DJO LLC [Chattanooga Rehab], Dallas, TX), which has been used in previous research exploring the effect of tDCS on emotional processing (Clarke et al., 2014). The cathodal (negative) electrode was placed on the left superior region of the trapezius muscle near the base of the participant's neck so that no stimulation was given to other brain regions. This reference electrode was kept in place by a rubber strap over the participants' shoulder.

tDCS stimulation for the active condition (tWMT) was fixed at $1.0 \mathrm{~mA}$ for $30 \mathrm{~min}$ of stimulation. Thus, charge density $\left(\mathrm{mA} / \mathrm{cm}^{2}\right)$ was fixed at $0.0387 \mathrm{~mA} / \mathrm{cm}^{2}$ in line with safety criteria for stimulation (Bikson et al., 2016; Nitsche et al., 2003). Stimulation began when participants initiated their first practice block and continued for $30 \mathrm{~min}$. Ramp-up time was approximately 30 seconds. Study staff was present for the duration of the stimulation and probed for discomfort or adverse events after each training block. For sham stimulation (sWMT), setup occurred the same way, but the stimulator was only turned on for the ramp-up portion (approximately 30 seconds) to induce a feeling of stimulation, and then 
turned off. Participants in the sham condition also received discomfort and safety monitoring between training blocks.

\section{Results}

\section{Demographic and Baseline Variables}

There were no significant group differences in terms of age or gender. No between group differences were observed on various measures at pretraining, including the ACS or DASS-21 (Table 1).

\section{Data Cleaning}

Response times for all cognitive tests were taken across individual participant's mean latencies for correct responses. Any trials that exceeded the participant's average response time by 2.5 standard deviations were deleted. Based on this data cleaning procedure, approximately $2.86 \%$ of trials were removed per person on average.

\section{Working Memory Performance During Training ( $n$-back Task) \\ The average $n$ from the $n$-back task was calculated for each participant across all four blocks. Results are shown in Table 2. An independent samples $t$ - test revealed no working memory differences in the average $n$ between groups. The average highest $n$ obtained across four blocks was $2.70(S D=0.50)$ and $2.80(S D=0.36)$ for tWMT and sWMT, respectively. The average $n$ obtained across four blocks for the tWMT condition was $1.92(S D=0.39)$, and SWMT was $2.08(S D=0.31)$.}

Table 2

Average $n$ achieved on the $n$-back working memory training task

\begin{tabular}{rcccc}
\hline & tWMT $M(S D)$ & sWMT $M(S D)$ & $t$ & 0 \\
\hline Block 1 & $1.64(0.30)$ & $1.80(0.40)$ & 1.44 & 0.16 \\
Block 2 & $2.04(0.50)$ & $2.15(0.33)$ & 0.86 & 0.40 \\
Block 3 & $2.00(0.46)$ & $2.16(0.36)$ & 1.36 & 0.18 \\
Block 4 & $2.00(0.46)$ & $2.20(0.37)$ & 1.53 & 0.14 \\
\hline
\end{tabular}

Note. tWMT $=$ working memory training plus active direct current stimulation; sWMT = working memory training plus sham direct current stimulation. ${ }^{*} p<.05 ;{ }^{* *} p<.01$

\section{Working Memory Performance after Training} We hypothesized that those in the tWMT group would improve WM performance on tasks of mathematical operations, reading, and symmetry relative to those in the sWMT group. A 2 (condition) $\times 2$ (time) repeated measures analysis of variance (ANOVA) was conducted to compare between group changes pre- and posttraining. The span tasks were analyzed using absolute and relative values as described in the methods section. These data are presented in Table 3.

The Operation Span task yielded significant positive main effects of time for absolute and relative scores, but no group by time interactions were found for either absolute or relative scores. The Reading Span task yielded no significant main effects of time for absolute or relative scores, or group by time interactions for absolute or relative scores. The Symmetry Span task yielded no significant main effects of time for absolute or relative scores, or group by time interactions for absolute or relative scores.
Cognitive Inhibition and Attentional Control

We predicted that those who received tWMT versus sWMT would improve related, untrained cognitive abilities in cognitive inhibition and attentional control. A 2 (condition) $\times 2$ (time) repeated measures ANOVA was conducted for the following cognitive measures.

Emotional Stroop. The EST was evaluated through two indices: (1) overall RT and (2) Stroop interference effects (bias). Results are shown in Table 4. There was a main effect of time, such that RT became shorter (i.e., faster reaction time) for neutral words, positive words, and negative words. No significant differences were observed for group $\mathrm{x}$ time interaction for differences in RT between groups on neutral words, positive words, or negative words. Finally, in terms of interference effects, no significant main effects of time were observed between groups for negative words or positive words. Further, no significant group $x$ time interaction effects were observed between group 
Table 3

Working memory performance before and after the computerized training program

\begin{tabular}{|c|c|c|c|c|c|c|c|c|c|}
\hline & & $\begin{array}{l}\text { tWMT } \\
M(S D)\end{array}$ & $\begin{array}{l}\text { sWMT } \\
M(S D)\end{array}$ & ME Time & $d$ & $95 \% \mathrm{Cl}$ & $\begin{array}{l}\text { Time } x \\
\text { Group }\end{array}$ & $d$ & $95 \% \mathrm{Cl}$ \\
\hline \multicolumn{10}{|c|}{ Operation Span } \\
\hline \multirow[t]{2}{*}{ Absolute } & Pre & $13.48(8.52)$ & $14.91(7.97)$ & \multirow{2}{*}{$\begin{array}{c}F(1,41)= \\
11.71, p<.01^{* *}\end{array}$} & \multirow{2}{*}{1.06} & \multirow{2}{*}[0.42,1.70]{} & \multirow{2}{*}{$\begin{array}{c}F(1,41)=0.57 \\
p=.46\end{array}$} & \multirow{2}{*}{0.20} & \multirow{2}{*}[-0.40,0.80]{} \\
\hline & Post & $16.38(7.42)$ & 19.45(8.90) & & & & & & \\
\hline Relative & Pre & $21.05(5.90)$ & $21.86(5.61)$ & $\begin{array}{c}F(1,41)=4.76 \\
p=.03^{*}\end{array}$ & 0.67 & {$[0.06,1.28]$} & $\begin{array}{c}F(1,41)=0.34 \\
p=.56\end{array}$ & 0.20 & {$[-0.40,0.80]$} \\
\hline \multirow[t]{2}{*}{ Absolute } & Pre & $9.48(7.93)$ & $12.23(8.29)$ & \multirow{2}{*}{$\begin{array}{c}F(1,41)=1.32 \\
p=.26\end{array}$} & \multirow{2}{*}{0.35} & \multirow{2}{*}[-0.25,0.95]{} & \multirow{2}{*}{$\begin{array}{c}F(1,41)=0.35 \\
p=.56\end{array}$} & \multirow{2}{*}{0.20} & \multirow{2}{*}[-0.40,0.80]{} \\
\hline & Post & $12.29(9.60)$ & $13.14(7.55)$ & & & & & & \\
\hline \multirow[t]{2}{*}{ Relative } & Pre & $18.38(6.45)$ & $21.55(5.84)$ & \multirow{2}{*}{$\begin{array}{c}F(1,41)=0.25 \\
p=.62\end{array}$} & \multirow{2}{*}{0.16} & \multirow{2}{*}[-0.43,0.76]{} & \multirow{2}{*}{$\begin{array}{c}F(1,41)=0.04 \\
p=.84\end{array}$} & \multirow{2}{*}{0.006} & \multirow{2}{*}[-0.59,0.60]{} \\
\hline & Post & $19.14(8.46)$ & $21.86(6.20)$ & & & & & & \\
\hline Relative & Pre & $13.90(5.99)$ & $14.43(5.21)$ & $F(1,40)=1.11$ & 035 & {$[-0$} & $F(1,40)=0.04$ & 006 & $7]$ \\
\hline & Post & $14.90(3.62)$ & $15.10(5.42)$ & $p=.30$ & 0.00 & {$[0.20,0.00]$} & $p=0.83$ & 0.00 & $0.01]$ \\
\hline
\end{tabular}

Note. 95\% confidence intervals (Cl) were calculated for associated effect size estimates $(d)$. tWMT = working memory training plus active direct current stimulation; sWMT = working memory training plus sham direct current stimulation. ${ }^{*} p<.05$; ${ }^{* *} p<.01$.

for negative words or positive words. Overall, while main effects of time were observed for overall RT from pre- to posttraining, no other main effect or group $\mathrm{x}$ time interaction differences were observed between groups.

Attention network task (ANT). The ANT was evaluated across three indices: alerting, orienting, and conflict. Results are found in Table 5 . No significant main effects of time were found for alerting, orienting, or conflict. There were also no significant group $\mathrm{x}$ time interactions for alerting, orienting, or conflict. Overall, no significant main effects of time or group $\mathrm{x}$ time interactions were observed for the ANT between groups.

\section{Conclusion}

WM is highly important in everyday cognitive functioning and has been proposed to be malleable through focused cognitive training programs (Jaeggi et al., 2008; Melby-Lervåg \& Hulme, 2013). tDCS has been proposed as a noninvasive means of increasing brain activation, which may in turn result in significant improvements in WM when paired with such training programs (Brunoni \& Vanderhasselt, 2014; Mancuso et al., 2016). Further, the use of emotional stimuli in WMT has been demonstrated to result in beneficial alterations in affect regulation as well as corresponding neural circuitry (Larsen et al., 2019; Schweizer et al., 2013, 2011). To this end, the present study sought to examine how a single session of tDCS-WMT, amplified with emotional valence, and dosage of training and electrical current, might improve WM capabilities and produce transfer effects on associated untrained cognitive processes. Performance across a range of WM domains was compared before and after WMT paired with active tDCS, versus sham tDCS. Contrary to study hypotheses, no group differences were found in the primary outcomes of working memory, and no group differences were found in untrained transfer abilities.

We did find that individuals in both training conditions evidenced faster reaction times on both the operation span task and the emotional Stroop task following the WMT session. The magnitude of these effects ranged from small to large and the direction of these effects suggested that individuals showed better cognitive performance over the course of the study overall. Considering that faster RT was observed in both groups, this change may 


\section{Table 4}

Interference control performance (Emotional Stroop Task) pre- and posttraining

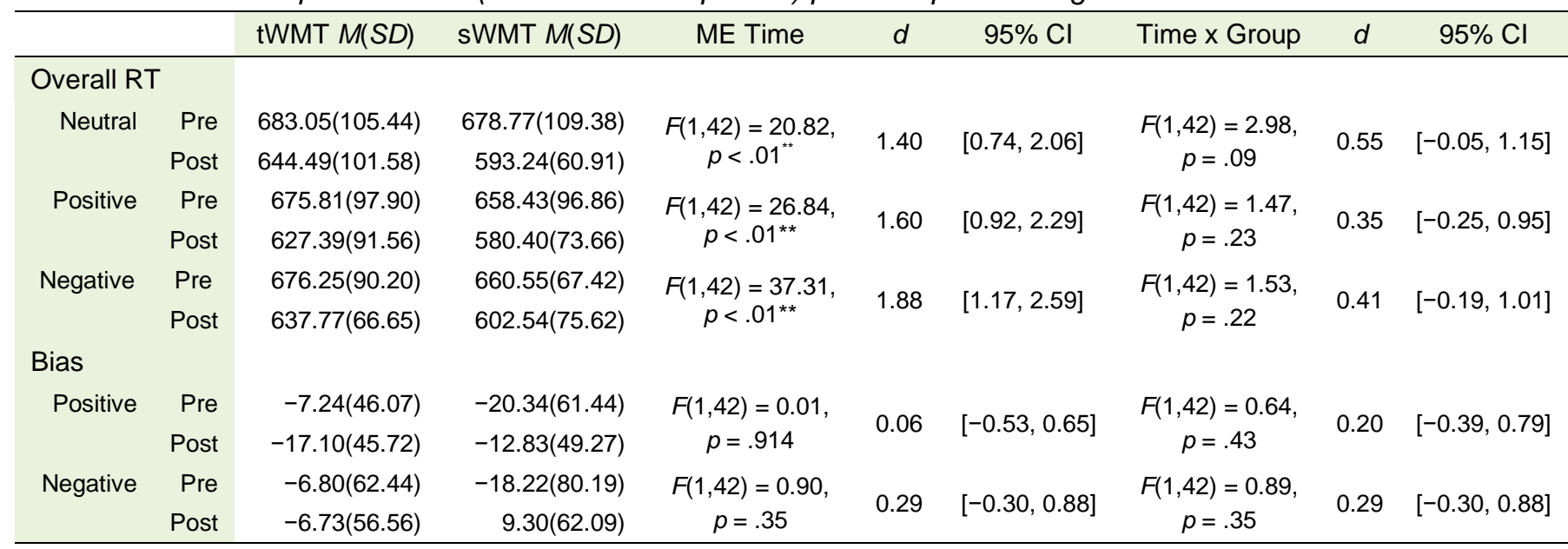

Note. 95\% confidence intervals $(\mathrm{Cl})$ were calculated for associated effect size estimates $(d)$. tWMT = working memory training plus active direct current stimulation; sWMT = working memory training plus sham direct current stimulation; RT = reaction time. ${ }^{\star} p<.05 ;{ }^{* *} p<.01$.

\section{Table 5}

Performance on attention control (Attention Network Tasks [ANT]) Pre- and Post-Training

\begin{tabular}{|c|c|c|c|c|c|c|c|c|c|}
\hline & & $\begin{array}{c}\text { tWMT } \\
\text { Mean }(S D)\end{array}$ & $\begin{array}{c}\text { sWMT } \\
\text { Mean }(S D)\end{array}$ & ME Time & $d$ & $95 \% \mathrm{Cl}$ & Time x Group & $d$ & $95 \% \mathrm{Cl}$ \\
\hline Alerting & Pre & 19.61(33.40) & $24.61(31.93)$ & $\begin{array}{c}F(1,41)=0.27 \\
p=.61\end{array}$ & 0.17 & {$[-0.43,0.77]$} & $\begin{array}{c}F(1,41)=0.00 \\
p=.98\end{array}$ & 0.06 & {$[-0.53,0.65]$} \\
\hline \multirow[t]{2}{*}{ Orienting } & Pre & $54.58(43.68)$ & $33.36(29.98)$ & \multirow{2}{*}{$\begin{array}{c}F(1,41)=0.04 \\
p=.84\end{array}$} & \multirow{2}{*}{0.06} & \multirow{2}{*}[-0.53,0.66]{} & \multirow{2}{*}{$\begin{array}{c}F(1,41)=2.09 \\
p=.16\end{array}$} & \multirow{2}{*}{0.46} & \multirow{2}{*}[-0.15,1.07]{} \\
\hline & Post & $46.63(32.25)$ & 43.97(19.49) & & & & & & \\
\hline
\end{tabular}

Note. 95\% confidence intervals $(\mathrm{Cl})$ were calculated for associated effect size estimates $(d)$. tWMT = working memory training plus active direct current stimulation; sWMT $=$ working memory training plus sham direct current stimulation. ${ }^{*} p<$ $.05 ;{ }^{* *} p<.01$.

be attributable to repeated assessment in a short window. Alternatively, faster RT on the operation span task and emotional Stroop may signal a reflection of improvements in efficiency in underlying memory cognitive network networks resulting from WMT. Previous research has suggested that WMT can indeed induce changes to produce faster reaction times (Thompson, Waskom, \& Gabrieli, 2016). Moreover, visuospatial working memory, which was a significant component in our $n$-back training, has been shown to be important in understanding variance in reaction time (Bo, Jennett, \& Seidler, 2011). Future research may benefit from more fine-grained analyses to explore how working memory, WMT paradigms, and reaction time distributions are connected.
There are likely numerous contributing factors to explain the lack of group differences between individuals who received active and sham tDCS. First and foremost, a single session of WMT, despite efforts to augment the strength of the training with emotional valence and tDCS, may not hold enough power to elicit significant change in the evaluated cognitive domains. Recent research suggests that the facilitative effects of WMT may be locked behind a dose-response relationship (Jaeggi \& Buschkuehl, 2014), and that transfer effects to untrained cognitive processes may be moderated by the duration of training (Schwaighofer, Fischer, \& Bühner, 2015). Deficient WM training effects may have yielded little room for augmentation effects by 
the tDCS manipulation, contributing to the lack of group differences.

Similarly, it is possible that the neurocognitive changes that follow tDCS are also subject to a doseresponse relationship. While some work has shown that a single session of tDCS can evoke change in cognitive performance in a pathological (e.g., depressed) sample, neurostimulation may function differently in the context of healthy controls (Gögler et al., 2017). Alonzo et al. (2012) found that daily tDCS was more effective in producing changes in cortical excitability than tDCS administered every other day. While it is also possible that we applied insufficient electrical current to induce changes to performance, previous research has suggested that stimulation in the range of $1.0 \mathrm{~mA}$ is optimal in WM protocols (Hoy et al., 2013).

It is also possible that we did not observe a significant effect of tDCS and WMT on working memory because of the lack of a follow-up visit. Indeed, some researchers have described so-called "sleeper effects" in which transfer effects of WMT for children and adolescents were only found months after training had been completed (Van der Molen, Van Luit, Van der Molen, Klugkist, \& Jongmans, 2010). While some researchers have highlighted the need for follow-up assessments (Holmes, Gathercole, \& Dunning, 2009), the present study involved a very short assessment time window; participants underwent pretraining testing, WMT and tDCS, and posttraining all in the span of a single lab visit which did not allow for any long-term delayed outcomes to be assessed. Individual differences such as preexisting cognitive abilities, motivation, enjoyment of cognitive challenges, beliefs about the malleability of intelligence have also been implicated for shorter-term WMT (Jaeggi, Buschkuehl, Shah, \& Jonides, 2014). It is also possible that WMT may not be as effective when presented in such concentrated format. The spacing effect, first identified by Ebbinghaus (2013/1885), posits that learning is most effective when spaced apart rather than completed in a short period of time. When considering the spacing effect alongside tDCS, researchers have shown that stimulation was more potent when spaced out over several days as compared to consecutive daily administration ( $\mathrm{Au}$, Buschkuehl, Duncan, \& Jaeggi, 2016). Transfer effects of tDCS to affective processes may also not be found when WMT is presented in such a short, concentrated session. Further still, extant work suggests that a single session of tDCS alone is not sufficient to produce noticeable cognitive gains (Horvath et al., 2015).
In light of the present study's results, the question arises of whether WMT is truly effective, a question that other researchers continue to debate. Some recent meta-analyses suggest that WMT has a small positive transfer effect on broader cognitive capabilities (Au et al., 2016; Karbach \& Verhaeghen, 2014). However, other researchers highlight some of the above considerations (e.g., discounting differences in baseline cognitive abilities, ignoring the use of active versus passive control groups) as problems in these meta-analyses, instead arguing there is a dearth of evidence in support of WMT (Melby-Lervåg \& Hulme, 2016). Some researchers have also found evidence for a publication bias in reporting significant effects of single-session tDCS studies (Westwood \& Romani, 2017). Rather than investigating whether or not WMT and tDCS themselves work, some argue that researchers should be more concerned with what parameters of training work best for which individuals, and how much improvement individuals make within WMT (Jaeggi, Buschkuehl, Jonides, \& Shah, 2011). Above and beyond WMT alone, other studies augmenting training with tDCS have found multiple sessions result in sustained gains in WM and untrained associated domains (Ruf et al., 2017). Again, perhaps the present study's single-session design limits the possibility of significant effects being found.

The findings of this study should be considered in light of its limitations, with those limitations shaping directions for future research. This study utilized a relatively small sample size (i.e., 22 participants per group), and it is possible that a larger sample size would have produced significant group differences. Granted, the estimated effect sizes for our variables were within their associated 95\% confidence internals; thus, it is also likely that additional data points would not have significantly altered the significance of findings.

While tDCS has been suggested to produce cognitive performance gains in a single session (Fregni et al., 2005), these claims have been disputed by other researchers (Alonzo et al., 2012; Meinzer et al., 2014). Future studies should consider multiple sessions of emotionally-laden WMT paired with tDCS. Finally, while this study compared active tDCS to sham tDCS, both groups completed an emotional $n$-back task. While the choice to use an emotional task was intentional given that tDCS has been implicated in improvements in affective and cognitive control, future studies might benefit from the inclusion of a 
neutral $n$-back task to investigate any potential group differences.

Despite largely null findings, the present study is methodologically sound and contributes to the newly growing body of tDCS literature. While our single session high-potency tDCS administration paired with an emotional $n$-back task did not result in significant changes across groups, the limitations addressed above provide future directions to explore within this area of work. As researchers continue to investigate the potential utility of WMT as a means of bolstering a wide range of cognitive functions and associated affective processes, it is imperative to explore mechanisms underlying these changes. For instance, are the positive changes resulting from WMT due to the brain working harder, or more efficiently? How far do possible transfer effects reach, and for how long? While our single session of WMT and tDCS administration did not result in any noticeable improvements or transfer effects, this allows researchers to narrow in on the minimal intervention needed to find such effects.

\section{Author Disclosure}

Authors have no grants, financial interests, or conflicts to disclose.

\section{References}

Alonzo, A., Brassil, J., Taylor, J. L., Martin, D., \& Loo, C. K. (2012). Daily transcranial direct current stimulation (tDCS) leads to greater increases in cortical excitability than second daily transcranial direct current stimulation. Brain Stimulation, 5(3), 208-213. https://doi.org/10.1016/j.brs.2011.04.006

Au, J., Buschkuehl, M., Duncan, G. J., \& Jaeggi, S. M. (2016). There is no convincing evidence that working memory training is NOT effective: A reply to Melby-Lervåg and Hulme (2015). Psychonomic Bulletin \& Review, 23(1), 331-337. https://doi.org/10.3758/s13423-015-0967-4

Baddeley, A. (1992). Working memory. Science, 255(5044), 556559. https://doi.org/10.1126/science.1736359

Barbey, A. K., Koenigs, M., \& Grafman, J. (2013). Dorsolateral prefrontal contributions to human working memory. Cortex, 49(5), $\quad$ 1195-1205. https://doi.org/10.1016 /j.cortex.2012.05.022

Beam, W., Borckardt, J. J., Reeves, S. T., \& George, M. S. (2009). An efficient and accurate new method for locating the F3 position for prefrontal TMS applications. Brain Stimulation, 2(1), 50-54. https://doi.org/10.1016/j.brs.2008.09.006

Beilock, S. L., \& Carr, T. H. (2005). When high-powered people fail: Working memory and "choking under pressure" in math. Psychological Science, 16(2), 101-105. https://doi.org /10.1111/j.0956-7976.2005.00789.x

Bikson, M., Grossman, P., Thomas, C., Zannou, A. L., Jiang, J., Adnan, T., ... Woods, A. J. (2016). Safety of transcranial direct current stimulation: Evidence based update 2016. Brain Stimulation, 9(5), 641-661. https://doi.org/10.1016 /j.brs.2016.06.004

Bo, J., Jennett, S., \& Seidler, R. D. (2011). Working memory capacity correlates with implicit serial reaction time task performance. Experimental Brain Research, 214(1), 73. https://doi.org/10.1007/s00221-011-2807-8
Brunoni, A. R., \& Vanderhasselt, M.-A. (2014). Working memory improvement with non-invasive brain stimulation of the dorsolateral prefrontal cortex: A systematic review and metaanalysis. Brain and Cognition, 86, 1-9. https://doi.org/10.1016 /j.bandc.2014.01.008

Clarke, P. J. F., Browning, M., Hammond, G., Notebaert, L., \& MacLeod, C. (2014). The causal role of the dorsolateral prefrontal cortex in the modification of attentional bias: Evidence from transcranial direct current stimulation. Biological Psychiatry, 76(12), 946-952. https://doi.org /10.1016/j.biopsych.2014.03.003

D'Esposito, M., \& Postle, B. R. (2015). The cognitive neuroscience of working memory. Annual Review of Psychology, 66, 115-142. https://doi.org/10.1146/annurevpsych-010814-015031

Dolcos, F., LaBar, K. S., \& Cabeza, R. (2004). Interaction between the amygdala and the medial temporal lobe memory system predicts better memory for emotional events. Neuron, 42(5), 855-863. https://doi.org/10.1016/S08966273(04)00289-2

Ebbinghaus, H. (2013). Memory: A contribution to experimental psychology. Annals of Neurosciences, 20(4), 155 https://doi.org/10.5214/ans.0972.7531.200408

Eriksson, J., Vogel, E. K., Lansner, A., Bergström, F., \& Nyberg, L. (2015). Neurocognitive architecture of working memory. Neuron, $\quad 88(1), \quad 33-46 . \quad$ https://doi.org/10.1016 /j.neuron.2015.09.020

Fan, J., McCandliss, B. D., Sommer, T., Raz, A., \& Posner, M. I. (2002). Testing the efficiency and independence of attentional networks. Journal of Cognitive Neuroscience, 14(3), 340-347. https://doi.org/10.1162/089892902317361886

Figueira, J. S. B., Oliveira, L., Pereira, M. G., Pacheco, L. B., Lobo, I., Motta-Ribeiro, G. C., \& David, I. A. (2017). An unpleasant emotional state reduces working memory capacity: Electrophysiological evidence. Social Cognitive and Affective Neuroscience, 12(6), 984-992. https://doi.org /10.1093/scan /nsx030

Filmer, H. L., Lyons, M., Mattingley, J. B., \& Dux, P. E. (2017). Anodal tDCS applied during multitasking training leads to transferable performance gains. Scientific Reports, 7(1), 12988. https://doi.org/10.1038/s41598-017-13075-y

Fregni, F., Boggio, P. S., Nitsche, M., Bermpohl, F., Antal, A., Feredoes, E., ... Pascual-Leone, A. (2005). Anodal transcranial direct current stimulation of prefrontal cortex enhances working memory. Experimental Brain Research, 166(1), 23-30. https://doi.org/10.1007/s00221-005-2334-6

Gögler, N., Willacker, L., Funk, J., Strube, W., Langgartner, S., Napiórkowski, N., ... Finke, K. (2017). Single-session transcranial direct current stimulation induces enduring enhancement of visual processing speed in patients with major depression. European Archives of Psychiatry and Clinical Neuroscience, 267(7), 671-686. https://doi.org /10.1007/s00406-016-0761-y

Hill, A. T., Fitzgerald, P. B., \& Hoy, K. E. (2016). Effects of anodal transcranial direct current stimulation on working memory: A systematic review and meta-analysis of findings from healthy and neuropsychiatric populations. Brain Stimulation, 9(2), 197-208. https://doi.org/10.1016/j.brs.2015.10.006

Holmes, J., Gathercole, S. E., \& Dunning, D. L. (2009). Adaptive training leads to sustained enhancement of poor working memory in children. Developmental Science, 12(4), F9-F15. https://doi.org/10.1111/j.1467-7687.2009.00848.x

Horvath, J. C., Forte, J. D., \& Carter, O. (2015). Quantitative review finds no evidence of cognitive effects in healthy populations from single-session transcranial direct current stimulation (tDCS). Brain Stimulation, 8(3), 535-550. https://doi.org/10.1016/j.brs.2015.01.400

Hoy, K. E., Emonson, M. R. L., Arnold, S. L., Thomson, R. H., Daskalakis, Z. J., \& Fitzgerald, P. B. (2013). Testing the limits: Investigating the effect of tDCS dose on working 
memory enhancement in healthy controls. Neuropsychologia, 51(9), 1777-1784. $\quad$ https://doi.org/10.1016 /j.neuropsychologia.2013.05.018

Hur, J., lordan, A. D., Dolcos, F., \& Berenbaum, H. (2017). Emotional influences on perception and working memory. Cognition and Emotion, 31(6), 1294-1302. https://doi.org $/ 10.1080 / 02699931.2016 .1213703$

Jaeggi, S. M., \& Buschkuehl, M. (2014). Working memory training and transfer: Theoretical and practical considerations. In B. Toni (Ed.), New Frontiers of Multidisciplinary Research in STEAM-H (Science, Technology, Engineering, Agriculture, Mathematics, and Health) (Vol 90, pp. 19-43). https://doi.org /10.1007/978-3-319-07755-0_2

Jaeggi, S. M., Buschkuehl, M., Jonides, J., \& Perrig, W. J. (2008). Improving fluid intelligence with training on working memory. Proceedings of the National Academy of Sciences of the United States of America, 105(19), 6829-6833. https://doi.org /10.1073/pnas.0801268105

Jaeggi, S. M., Buschkuehl, M., Jonides, J., \& Shah, P. (2011). Short- and long-term benefits of cognitive training. Proceedings of the National Academy of Sciences, 108(25), 10081-10086. https://doi.org/10.1073/pnas.1103228108

Jaeggi, S. M., Buschkuehl, M., Shah, P., \& Jonides, J. (2014). The role of individual differences in cognitive training and transfer. Memory \& Cognition, 42(3), 464-480. https://doi.org/10.3758/s13421-013-0364-z

Jaeggi, S. M., Studer-Luethi, B., Buschkuehl, M., Su, Y.-F., Jonides, J., \& Perrig, W. J. (2010). The relationship between $n$-back performance and matrix reasoning-implications for training and transfer. Intelligence, 38(6), 625-635. https://doi.org/10.1016/j.intell.2010.09.001

Karbach, J., \& Verhaeghen, P. (2014). Making working memory work: A meta-analysis of executive-control and working memory training in older adults. Psychological Science, 25(11), 2027-2037. https://doi.org/10.1177 10956797614548725

Larsen, S. E., Lotfi, S., Bennett, K. P., Larson, C. L., DeanBernhoft, C., \& Lee, H.-J. (2019). A pilot randomized trial of a dual $n$-back emotional working memory training program for veterans with elevated PTSD symptoms. Psychiatry Research, 275, 261-268. https://doi.org/10.1016 /j.psychres.2019.02.015

Lovibond, P. F., \& Lovibond, S. H. (1995). The structure of negative emotional states: Comparison of the Depression Anxiety Stress Scales (DASS) with the Beck Depression and Anxiety Inventories. Behaviour Research and Therapy, 33(3), 335-343. https://doi.org/10.1016/0005-7967(94)00075-U

Lukasik, K. M., Waris, O., Soveri, A., Lehtonen, M., \& Laine, M. (2019). The relationship of anxiety and stress with working memory performance in a large non-depressed sample. Frontiers in Psychology, 10, 4. https://doi.org/10.3389 /fpsyg.2019.00004

Mancuso, L. E., Ilieva, I. P., Hamilton, R. H., \& Farah, M. J. (2016). Does transcranial direct current stimulation improve healthy working memory?: A meta-analytic review. Journal of Cognitive Neuroscience, 28(8), 1063-1089. https://doi.org /10.1162/jocn_a_00956

Martin, D. M., Teng, J. Z., Lo, T. Y., Alonzo, A., Goh, T., lacoviello, B. M., ... Loo, C. K. (2018). Clinical pilot study of transcranial direct current stimulation combined with Cognitive Emotional Training for medication resistant depression. Journal of Affective Disorders, 232, 89-95. https://doi.org/10.1016/j.jad.2018.02.021

Matsuo, K., Glahn, D. C., Peluso, M. A. M., Hatch, J. P., Monkul, E. S., Najt, P., ... Soares, J. C. (2007). Prefrontal hyperactivation during working memory task in untreated individuals with major depressive disorder. Molecular Psychiatry, 12(2), 158-166. https://doi.org/10.1038 /sj.mp.4001894
Meinzer, M., Jähnigen, S., Copland, D. A., Darkow, R., Grittner, U., Avirame, K., ... Flöel, A. (2014). Transcranial direct current stimulation over multiple days improves learning and maintenance of a novel vocabulary. Cortex, 50, 137-147. https://doi.org/10.1016/j.cortex.2013.07.013

Melby-Lervåg, M., \& Hulme, C. (2013). Is working memory training effective? A meta-analytic review. Developmental Psychology, 49(2), 270-291. https://doi.org/10.1037 la0028228

Melby-Lervåg, M., \& Hulme, C. (2016). There is no convincing evidence that working memory training is effective: A reply to $\mathrm{Au}$ et al. (2014) and Karbach and Verhaeghen (2014). Psychonomic Bulletin \& Review, 23(1), 324-330. https://doi.org/10.3758/s13423-015-0862-z

Moon, C.-M., \& Jeong, G.-W. (2015). Functional neuroanatomy on the working memory under emotional distraction in patients with generalized anxiety disorder. Psychiatry and Clinical Neurosciences, 69(10), 609-619. https://doi.org /10.1111/pcn.12295

Moran, T. P. (2016). Anxiety and working memory capacity: A meta-analysis and narrative review. Psychological Bulletin, 142(8), 831-864. https://doi.org/10.1037/bul0000051

Nitsche, M. A., Fricke, K., Henschke, U., Schlitterlau, A., Liebetanz, D., Lang, N., ... Paulus, W. (2003) Pharmacological modulation of cortical excitability shifts induced by transcranial direct current stimulation in humans. The Journal of Physiology, 553(1), 293-301. https://doi.org /10.1113/jphysiol.2003.049916

Oswald, F. L., McAbee, S. T., Redick, T. S., \& Hambrick, D. Z (2015). The development of a short domain-general measure of working memory capacity. Behavior Research Methods, 47(4), 1343-1355. https://doi.org/10.3758/s13428-014-0543-2

Owens, M., Koster, E. H. W., \& Derakshan, N. (2013). Improving attention control in dysphoria through cognitive training: Transfer effects on working memory capacity and filtering efficiency. Psychophysiology, 50(3), 297-307. https://doi.org /10.1111/psyp.12010

Plewnia, C., Schroeder, P. A., Kunze, R., Faehling, F., \& Wolkenstein, L. (2015). Keep calm and carry on: Improved frustration tolerance and processing speed by transcranial direct current stimulation (tDCS). PLOS ONE, 10(4). https://doi.org/10.1371/journal.pone.0122578

Ruf, S. P., Fallgatter, A. J., \& Plewnia, C. (2017). Augmentation of working memory training by transcranial direct current stimulation (tDCS). Scientific Reports, 7(1), 876. https://doi.org/10.1038/s41598-017-01055-1

Salazar-Villanea, M., Liebmann, E., Garnier-Villarreal, M., Montenegro-Montenegro, E., \& Johnson, D. K. (2015). Depressive symptoms affect working memory in healthy older adult hispanics. Journal of Depression \& Anxiety, 4(4). https://doi.org/10.4172/2167-1044.1000204

Sari, B. A., Koster, E. H. W., Pourtois, G., \& Derakshan, N. (2016). Training working memory to improve attentional control in anxiety: A proof-of-principle study using behavioral and electrophysiological measures. Biological Psychology, 121, 203-212. https://doi.org/10.1016 /j.biopsycho.2015.09.008

Schmidt, M. E., Wolkenstein, L., \& Plewnia, C. (2015). P165. Effects of transcranial direct current stimulation on training cognitive control over emotional distraction. Clinical Neurophysiology, 126(8), e142. https://doi.org/10.1016 /j.clinph.2015.04.230

Schulze, L., Grove, M., Tamm, S., Renneberg, B., \& Roepke, S. (2019). Effects of transcranial direct current stimulation on the cognitive control of negative stimuli in borderline personality disorder. Scientific Reports, 9(1), 332. https://doi.org/10.1038 Is41598-018-37315-x

Schwaighofer, M., Fischer, F., \& Bühner, M. (2015). Does working memory training transfer? A meta-analysis including training 
conditions as moderators. Educational Psychologist, 50(2), 138-166. https://doi.org/10.1080/00461520.2015.1036274

Schweizer, S., Grahn, J., Hampshire, A., Mobbs, D., \& Dalgleish, T. (2013). Training the emotional brain: Improving affective control through emotional working memory training. Journal of Neuroscience, 33(12), 5301-5311. https://doi.org/10.1523 /JNEUROSCI.2593-12.2013

Schweizer, S., Hampshire, A., \& Dalgleish, T. (2011). Extending brain-training to the affective domain: Increasing cognitive and affective executive control through emotional working memory training. PLOS ONE, 6(9), e24372. https://doi.org /10.1371/journal.pone.0024372

Schweizer, S., Samimi, Z., Hasani, J., Moradi, A., Mirdoraghi, F., \& Khaleghi, M. (2017). Improving cognitive control in adolescents with post-traumatic stress disorder (PTSD). Behaviour Research and Therapy, 93, 88-94. https://doi.org /10.1016/j.brat.2017.03.017

Smith, P., \& Waterman, M. (2003). Processing bias for aggression words in forensic and nonforensic samples. Cognition and Emotion, 17(5), 681-701. https://doi.org/10.1080 /02699930302281

Soveri, A., Antfolk, J., Karlsson, L., Salo, B., \& Laine, M. (2017). Working memory training revisited: A multi-level metaanalysis of $n$-back training studies. Psychonomic Bulletin \& Review, 24(4), 1077-1096. https://doi.org/10.3758/s13423016-1217-0

Stout, D. M., Shackman, A. J., Johnson, J. S., \& Larson, C. L. (2015). Worry is associated with impaired gating of threat from working memory. Emotion, 15(1), 6-11. https://doi.org /10.1037/emo0000015

Stout, D. M., Shackman, A. J., \& Larson, C. L. (2013). Failure to filter: Anxious individuals show inefficient gating of threat from working memory. Frontiers in Human Neuroscience, 7. https://doi.org/10.3389/fnhum.2013.00058

Thompson, T. W., Waskom, M. L., \& Gabrieli, J. D. E. (2016). Intensive working memory training produces functional changes in large-scale frontoparietal networks. Journal of Cognitive Neuroscience, 28(4), 575-588. https://doi.org /10.1162/jocn_a_00916

Van der Molen, M. J., Van Luit, J. E. H., Van der Molen, M. W., Klugkist, I., \& Jongmans, M. J. (2010). Effectiveness of a computerised working memory training in adolescents with mild to borderline intellectual disabilities. Journal of Intellectual Disability Research: JIDR, 54(5), 433-447. https://doi.org/10.1111/j.1365-2788.2010.01285.x

Westwood, S. J., \& Romani, C. (2017). Transcranial direct current stimulation (tDCS) modulation of picture naming and word reading: A meta-analysis of single session tDCS applied to healthy participants. Neuropsychologia, 104, 234-249. https://doi.org/10.1016/j.neuropsychologia.2017.07.031

Received: April 17, 2020

Accepted: August 18, 2020

Published: September 30, 2020 